\title{
Accuracy of surface registration in cranial electromagnetic navigation
}

\author{
Annette Runge ${ }^{1 *}$, Teresa Steinbichler ${ }^{1}$, Aristeidis Giotakis ${ }^{1}$, Volker Schartinger ${ }^{1}$, Romed Hörmann ${ }^{2}$, Herbert Riechelmann ${ }^{1}$ and Wolfgang \\ Freysinger ${ }^{1}$ \\ ${ }^{1}$ Department of Otolaryngology- Head and Neck Surgery, Medical University of Innsbruck, Austria \\ ${ }^{2}$ Department for Anatomy, Histology and Embryology, Medical University of Innsbruck, Austria
}

\begin{abstract}
Purpose: We evaluated the accuracy of surface registration with and without additional landmark registration during cranial electromagnetic navigation. Results were referenced to point by point registration with bone anchored fiducials.

Methods: Using a commercially available electromagnetic navigation system, 4 rhinologic surgeons applied surface registration alone (SurfReg) and surface registration with additional anatomic landmarks (CombReg) in a macerated human skull. Point by point registration using bone anchored fiducials (RefReg) served as reference. Target registration error (TRE) served as a measure for accuracy. For TRE calculation, 16 landmarks on the viscerocranium, the frontal skull base and the middle cranial fossa were touched with a probe and the distance of the probe and the target was measured on a screenshot of the corresponding CT image in three planes. A mixed regression model with registration mode as fixed factor and examiner and target as random factors was used.
\end{abstract}

Results: Mean (95\% CI) TRE for SurfReg was 1.12 (0.86 to 1.39) $\mathrm{mm}$ and 1.21 (0.94 to 1.47) mm for CombReg (p=0.13). TRE of the RefReg was significantly lower $(0.91 \mathrm{~mm} ; 0.64$ to $1.18 ; \mathrm{p}<0.001)$. TRE varied significantly depending on target screw position. TRE did not vary significantly among different examiners.

Conclusion: Surface registration is considered sufficiently accurate for clinical application during FESS and frontal skull base surgery. Additional landmarks did not improve accuracy when compared with surface registration alone. Additional time for landmark registration can therefore be spared.

\section{Introduction}

Electromagnetic surgical navigation systems improve and facilitate orientation in difficult anatomic settings such as the paranasal sinuses and the anterior skull base. The surgeon can move the instruments freely without dependence on an uninterrupted line of sight [1-3]. Electromagnetic navigation may replace optical navigation, which according to some studies yields better tracking accuracy $[2,4]$, because algorithms have been developed to reduce the interference of ferromagnetic objects within the magnetic transmitter field and to improve accuracy in electromagnetic navigation $[5,6]$. The key to accurate intraoperative navigation is registration, i.e. aligning preoperative radiologic images with the patient's anatomy at the current position in the surgical theatre [7]. Depending on the kind of reference used, patient registration may be subdivided in three groups. Headframes are applied for neurosurgical and neuroradiological interventions and fixate the patient's head as well as the instruments for accurate treatment without damage of surrounding tissue [8]. Point by point registration uses anatomical landmarks or fiducial markers attached to bone or skin. A targeting device, for instance a pointer, is used to measure positions of markers while its position is tracked by a computer system that also holds the image data. After registration the position of the device is displayed in image data on the computer screen [9]. In general, fiducials provide better accuracy than anatomical landmarks [10]. Skin adhesive fiducials are subject to skin shift intraoperatively. Application of bone anchored fiducials is invasive. Both bone anchored, and skin adhesive fiducials require additional CT scans preoperatively. The third mode of registration is surface registration where the patient's face is digitized with a $3 \mathrm{D}$ tracker and registered to the preoperative state $3 \mathrm{D}$ surface
$[7,11]$. Like point by point registration using anatomical landmarks, surface registration does not require additional radiologic imaging and is noninvasive.

Target Registration Error (TRE) is the pertinent measure for tracking accuracy [12]. It defines the distance between the landmark on the CT dataset and the coordinates indicated by the tracking system when pointing to the corresponding landmark which was not used for registration [13,14]. Current data comparing different registration modes in electromagnetic tracking suggest that point by point registration using bone anchored fiducials results in lowest TRE, i.e. best accuracy $[15,16]$. Some surgical navigation systems offer the possibility to use both, surface registration and in addition point by point landmark registration in the same run [16]. In this study we investigated if combined surface and point by point landmark registration (CombReg) provides better accuracy than surface registration alone (SurfReg) at the paranasal sinuses and the frontal skull base. Point by point registration with bone anchored fiducial markers (RefReg) served as a reference.

${ }^{\star}$ Correspondence to: Annette Runge, Department of Otolaryngology-Head and Neck Surgery, Medical University of Innsbruck, Anichstr, 356020 Innsbruck, Austria, Tel: 0043 05050423142; Email: annette.runge@tirol-kliniken.at

Key words: computer assisted surgery, natural orifice endoscopic surgery, electromagnetic fields, data measurement accuracy

Received: April 05, 2018; Accepted: April 20, 2018; Published: April 23, 2018 


\section{Material and methods}

For this experimental study, a macerated human skull was provided by the Department of Anatomy, Medical University of Innsbruck. To obtain access to the frontal skull base, the calvarium was partly removed. Altogether, 16 prominent fiducial sites of the viscerocranium and the anterior skull base were marked with titanium screws measuring 1 $\mathrm{mm}$ in diameter (Leibinger, Freiburg, Germany; Table 1). A CT scan with a slice thickness of $1 \mathrm{~mm}$ (Somatom Definition Flash, Siemens, Erlangen, Germany) was performed and image data were transferred to the navigation system's internal storage (Stealth System S7, AxiEM System, Medtronic, Louisville, CO, USA).

\section{Set up}

All experiments were performed in the surgical theatre to account for possible electromagnetic disturbances. The skull was placed on an operating table. The electromagnetic navigation system was set up as done during endoscopic skull base surgery. The magnetic field emitter was fixed to the operating table within close proximity to the skull. A self-adhesive dynamic reference frame was attached to the forehead and a non-ferromagnetic pointer was supplied with an electromagnetic sensor. Both appliances were connected to the electromagnetic control unit. All program applications and CT data could be viewed on a monitor opposing the surgeon.

\section{Study design}

Four ENT surgeons applied 3 registration methods in a random order: surface registration alone (SurfReg), registration of both anatomical landmarks and surface (CombReg) and registration of bone anchored fiducials as the reference method (RefReg). For SurfReg, the surgeon traced the outlines of the orbital rims and the nose using the tracked pointer. During CombReg, the nasion, the anterior nasal spine and the left supraorbital foramen served as landmarks in addition to surface registration. For registration with bone anchored fiducial markers (RefReg), six fiducials were manually marked on the CT image and then identified on the skull with the registration probe. Fiducials included screws in at the nasofrontal suture, the anterior nasal spine as well as the screw pairs on the frontal processes of the maxilla and zygomaticofrontal sutures. RefReg was complete when the predicted fiducial registration error was estimated less than $1.2 \mathrm{~mm}$ by the navigation system [17].

To assess operator related variability of registration, each of the 4 ENT-surgeons replicated the whole process of registration with each registration and measured each of the 16 targets mode 10 times. For TRE measurements, the deviation of the tip of the pointer from actual fiducial sites was measured. This was accomplished by touching the center of the titanium screws with the registration probe and taking a screen shot of the corresponding CT images. Distance between the actual screw center and navigated position of the pointer was measured in axial, coronal and sagittal planes. Thus, for each landmark, measurements in 3 dimensions (anterior/posterior; left/right and superior/inferior) were taken to calculate the Target Registration Error (TRE) (Figure 1).

\section{Data analysis}

Raw data were the deviations of the tracer position from the target screw in the 3 orthogonal planes in millimeters. Deviations to the right, to posterior and to superior were coded positively, deviations to the left, to anterior and to inferior were coded negatively. The Euclidean
Table 1: Average TRE depending on registration method. displays the average Target Registration Error (TRE) for each registration method calculated from all individual measurements performed after each course of registration.

\begin{tabular}{|c|c|c|c|c|}
\hline Registration & Mean & Standard error & $\begin{array}{c}\mathbf{9 5 \%} \\
\text { Confidence } \\
\text { interval } \\
\text { Lower Bound }\end{array}$ & $\begin{array}{c}\mathbf{9 5 \%} \\
\text { Confidence } \\
\text { interval } \\
\text { Upper bound }\end{array}$ \\
\hline SurfReg & 1.12 & 0.11 & 0.86 & 1.39 \\
\hline CombReg & 1.21 & 0.11 & 0.94 & 1.47 \\
\hline RefReg & 0.91 & 0.11 & 0.64 & 1.18 \\
\hline
\end{tabular}

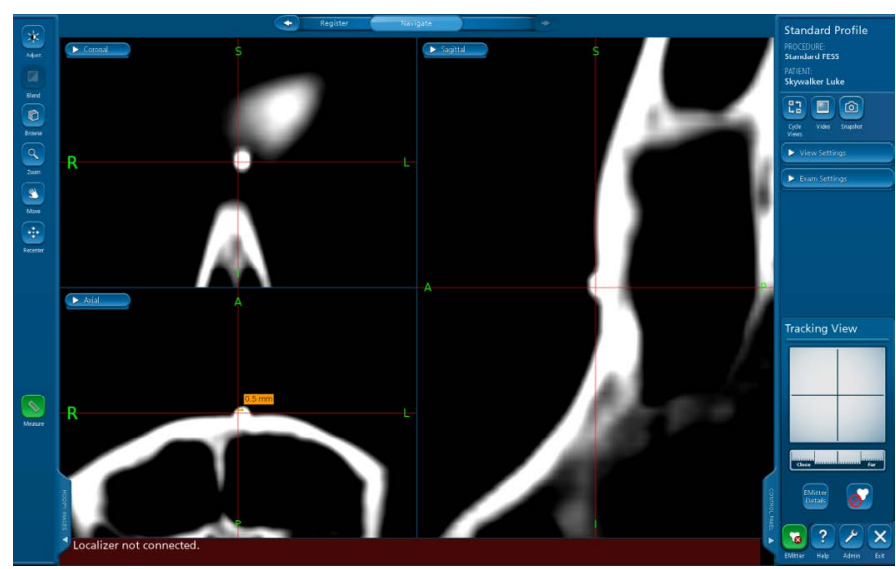

Figure 1: A screenshot was taken by switching off the electromagnetic emitter after touching a screw. The distance between the actual center of the screw head and the navigated position of the pointer, visible as the center of the red coordinate system, were measured manually by the examiner.

distances in the 3 planes were calculated and taken as the TRE. In a next step, the measurement repeats of each registration were averaged to obtain 1 TRE value per user, target screw and registration mode. Coefficients of variation in percent were calculated to estimate operator related variability. Finally, TRE was used as the outcome variable in a linear mixed model with registration mode as a fixed effect. Target screw and operator were included as random effects. Variance components was used as covariance structure. Restricted maximum likelihood was used for calculations [18].

\section{Results}

\section{TRE and registration mode}

Mean TRE (95\% CI) was 1.12 (0.86 to $1.39 \mathrm{~mm})$ with SurfReg and $1.21 \mathrm{~mm}$ (0.94 to $1.47 \mathrm{~mm}$ ) using CombReg ( $\mathrm{p}=0.13$; Table 1$)$. TRE of the reference method (RefReg using fiducials) was significantly smaller ( $0.91 \mathrm{~mm}$; $95 \%$ CI 0.64 to $1.18 \mathrm{~mm}$ ) than TRE of the 2 non-fiducial registration modes $(\mathrm{p}<0.001)$. In the applied mixed model, random effects caused a residual TRE variance of $0.17 \mathrm{~mm}$. The variance differed significantly from zero for the 16 target screws ( $28 \%$ of total variance; $\mathrm{p}=0.02$ ), whereas TRE variance for the 4 operators was not significantly different from zero ( $18 \%$ of total variance; $\mathrm{p}=0.25$ ).

\section{TRE and target position}

Using target screw position as a fixed factor and operator and registration mode as random factors, TRE differed significantly depending on target screw position $(\mathrm{p}<0.001)$. The smallest TRE was observed at the nasofrontal suture $(0.61 \mathrm{~mm})$, the highest at the left zygomatic arch $(2.11 \mathrm{~mm})$ (Table 2; Figure 2). TRE variance of target screw position did not significantly differ from zero for operator $(\mathrm{p}=0.25)$ and for registration mode $(\mathrm{p}=0.35)$. 
Table 2: Mean TRE at the 16 target screws, displays the Target Registration Error (TRE) measured at 16 fiducial sites using surface registration (SurfReg) and surface registration in combination of anatomical landmarks (CombReg). In addition. the reference method. registration of bone anchored fiducials (RefReg) was performed. All fiducial sites mentioned in the first column were marked by titanium screws.

\begin{tabular}{|l|c|c|c|}
\hline Measuring point & TRE SurfReg & TRE CombReg & TRE RefReg \\
\hline right zygomaticofrontal suture & 0.78 & 0.91 & 0.94 \\
\hline left zygomaticofrontal suture & 0.78 & 0.97 & 0.71 \\
\hline nasofrontal suture & 0.61 & 0.78 & 0.76 \\
\hline anterior nasal spine & 1.31 & 1.15 & 1.04 \\
\hline right frontal process of maxilla & 1.03 & 0.93 & 0.6 \\
\hline left frontal process of maxilla & 1.18 & 1.24 & 0.75 \\
\hline right lesser wing of sphenoid & 1.07 & 0.92 & 0.98 \\
\hline left lesser wing of sphenoid & 1.1 & 0.86 & 0.94 \\
\hline right zygomatic arch & 1.46 & 1.43 & 1.35 \\
\hline left zygomatic arch & 1.47 & 2.11 & 1.3 \\
\hline floor of sphenoid sinus & 1.05 & 1.38 & 1.13 \\
\hline right posterior wall of frontal sinus & 1.31 & 1.69 & 0.84 \\
\hline left posterior wall of frontal sinus & 1.1 & 1.29 & 0.8 \\
\hline right cribriform plate & 1.29 & 1.26 & 0.75 \\
\hline left cribriform plate & 1.08 & 1.03 & 0.9 \\
\hline fossa of pituitary gland & 1.51 & 1.46 & 0.77 \\
\hline 1)Reference method & & & \\
\hline
\end{tabular}

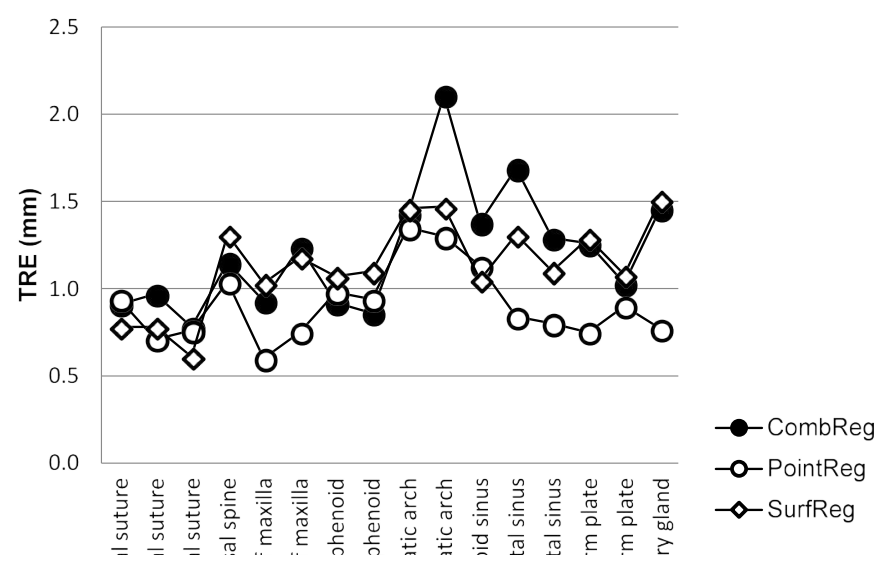

Figure 2: The $\mathrm{x}$ - axis lists the landmarks by ascending screw numbers, the $\mathrm{y}$ - axis contains the Target Registration Error of each measuring point in $\mathrm{mm}$. Each line depicts one registration mode (see labeling legend).

To analyze, if posterior or lateral targets have lower TRE than anterior and medial targets, the distances from the facial plane (anterior/ posterior) and from the mid-plane (left/right) were ranked. TRE of corresponding lateral targets of the right and left side were averaged resulting in 10 distance ranks. No correlation of ranked distances from the facial-plane or the mid-plane and TRE was observed.

\section{Variability of repeated registrations}

All measurements were performed in 10 replicates. For each replicate, the whole process of registration and subsequent TRE determination was performed by each of the 4 operators. Coefficient of variation in percent $(\mathrm{CV})$ was used to express variability of replicated measurements. For CV analysis, a mixed model was used just as described for TRE analysis. Overall, mean CV was 38\% (95\% CI $27 \%$ to $50 \%)$. Registration mode $(\mathrm{p}>0.2)$ and target position $(\mathrm{p}=0.13)$ had no influence on $\mathrm{CV}$.

\section{Discussion}

We studied surface-based registration modes with and without additional registration of anatomical landmarks in electromagnetic tracking at the paranasal sinuses and frontal skull base. Surface based registration modes reflect common clinical practice, mainly because it does not require fiducial positioning before preoperative image acquisition. Consequently, routine computer tomography performed for preoperative diagnosis suffices as image data for intraoperative navigation. Surface registration can be supplemented by point by point registration using anatomical landmarks aiming to improve navigation accuracy. Anatomical landmarks are also defined without pre-CT fiducial positioning. Target registration error (TRE) served as outcome parameter for accuracy $[19,20]$. Position of 16 targets and performance of 4 operators were included as random factors.

\section{Addition of landmark registration to surface registration did not improve accuracy}

We observed a mean TRE of $1.12 \mathrm{~mm}$ for SurfReg and $1.21 \mathrm{~mm}$ for CombReg $(\mathrm{p}=0.13)$. This means that addition of landmarks to surface registration did not improve accuracy of surgical navigation. Overall, the TRE values observed in this study were within the range reported in other studies of electromagnetic registration [13,15,21-24]. We were also interested, how accuracy of surface registration compares to the reference registration mode using bone anchored fiducials applied before the preoperative CT scan. Surface registration without fiducials yielded significantly worse TRE values than the reference method $(0.91$ $\mathrm{mm} ; \mathrm{p}<0.001)$. However, the advantages of surface registration justify, in our opinion, this low loss of accuracy. The observed difference in navigation accuracy of $0.2-0.3 \mathrm{~mm}$ was considerably lower than previously reported. Soteriou et al. [22] compared several registration modes, including bone anchored fiducial markers, surface registration and anatomic landmarks. Point by point registration using bone anchored fiducials resulted in highest accuracy (TRE $=0.94 \pm 0.06 \mathrm{~mm}$ ), whereas surface registration gained an approx. 70\% higher TRE (1.59 $\pm 0.14 \mathrm{~mm}$ ), and registration of anatomic landmarks resulted in an up to $346 \%$ higher mean TRE $(4.2 \pm 0.73)$. Ledderose et al. [25] observed a mean TRE of $0.33 \mathrm{~mm}$ for registration with bone anchored fiducials, whereas surface registration resulted in a higher mean TRE of $1.9 \mathrm{~mm}$. (approx. $475 \%$ worse). A difference of 1.7 vs. $4.0 \mathrm{~mm}$ (135\%) between bone anchored fiducials and surface registration was found during intracranial navigation by Mascott et al. [26].

\section{Sources of error}

There are certain potential sources of error during registration regardless of registration mode. One potential source of error is reproducibility of patient registration. A coefficient of variation of TRE of almost $40 \%$ in 10 repeats of the registration process of each individual user is considered a substantial variability. In contrast, variance of TRE among users was not significant, meaning that no individual user outperformed the others.

Another potential source of error is the target distance from the facial plane and mid-plane. An anterior/posterior and left/right ranking did not bring forth any correlation with TRE. This is in contrast with some other studies, where an increase in TRE was observed when targeting more posteriorly located fiducial sites [13], whereas Komune et al. [15] found no difference in TRE depending on the location in lateral skull base surgery. Possible reasons for these diverging results may be the use of different navigation systems as well as varying distances between the fiducial target sites and the reference frame among the various study groups. However, the least mean TRE was generated at the nasofrontal 
suture $(0.61 \mathrm{~mm})$, followed by anatomic structures of the orbital conus, structures, which are close to the area of registration, as supported by the findings of Ahmadian et al. [24] and Li et al. [27]. The largest TRE was measured on both zygomatic arches $(1.41$ and $2.11 \mathrm{~mm})$. Those landmarks were at the most lateral positions in the electromagnetic field, where accuracy in electromagnetic tracking has been shown to be weak $[28,29]$.

\section{User localization and fiducial localization error}

User Localization Error is the pure user error of physically placing the probe into fiducials or anatomic landmarks [17]. Anatomical structures or fiducial sites may have been confused with bony structures during measurements when the target was not clearly visible in all planes as described by Chang and coworkers [30]. Human factors influence accuracy during pointing for registration, such as muscular fatigue resulting in increased positioning tremor [31,32]. With or without fiducial markers, misidentification can occur using surgical navigation systems. Shift or even dislocation of the reference frame may occur, with resulting loss of accuracy correlation with an increasing time span between registration and application of navigation. [33]. The fiducial localization error (FLE) describes the error made when localizing loci for registration in image space and on the patient. The pointer was not always aimed at the center of the screw head at an exact $90^{\circ}$ angle. A wider surface contact and an eccentric location of the actual instrument's tip may result in loss of pointing accuracy [20].

\section{Conclusion}

The absolute values of mean TRE of both, SurfReg and CombReg suggest clinically practicable registration accuracy when compared to the much more invasive reference method. As there is no accuracy improvement adding anatomical landmark registration to surface registration, this additional effort may be spared. If optimal accuracy is mandatory, the surgeon should consider implanted fiducial markers and optical navigation.

\section{References}

1. Hahn P, Oezdemir S, Komp M, Giannakopoulos A, Kasch R, et al. (2015) Navigation of pedicle screws in the thoracic spine with a new electromagnetic navigation system: a human cadaver study. Biomed Res Int 2015: 183586. [Crossref]

2. Kral F, Puschban EJ, Riechelmann H, Pedross F, Freysinger W (2011) Optical and electromagnetic tracking for navigated surgery of the sinuses and frontal skull base. Rhinology 49: 364-368. [Crossref]

3. Freysinger C (2003) Computer assistance for intraoperative navigation in ENT surgery. Minim Invasive Ther Allied Technol 12: 36-51. [Crossref]

4. Kral F, Puschban EJ, Riechelmann H, Freysinger W (2013) Comparison of optical and electromagnetic tracking for navigated lateral skull base surgery. Int J Med Robot 9: 247-252. [Crossref]

5. Li M, Bien T, Rose G (2014) Construction of a conductive distortion reduced electromagnetic tracking system for computer assisted image-guided interventions. Med Eng Phys 36: 1496-1501. [Crossref]

6. Bien T, Li M, Salah Z, Rose G (2014) Electromagnetic tracking system with reduced distortion using quadratic excitation. Int J Comput Assist Radiol Surg 9: 323-332. [Crossref]

7. Kristin J, Mucha D, Schipper J, Klenzner T (2012) Registration strategies for the application of the navigation system Fiagon at the lateral scull base. Laryngorhinootologie 91: 306-310. [Crossref]

8. Safaee M, Burke J, McDermott MW (2016) Techniques for the Application of Stereotactic Head Frames Based on a 25-Year Experience. Cureus 8: e543. [Crossref]

9. Fitzpatrick JM, West JB, Maurer CR Jr (1998) Predicting error in rigid-body pointbased registration. IEEE Trans Med Imaging 17: 694-702. [Crossref]
10. Grauvogel TD, Soteriou E, Metzger MC, Berlis A, Maier W (2010) Influence of different registration modalities on navigation accuracy in ear, nose, and throat surgery depending on the surgical field. Laryngoscope 120: 881-888. [Crossref]

11. Besl PJ MN (1992) A Method for Registration of 3-D Shapes. IEEE Transactions on Pattern Analysis AND Machine Intelligence 14: 239-256.

12. Fitzpatrick JM (2010) The role of registration in accurate surgical guidance. Proc Inst Mech Eng H 224: 607-622. [Crossref]

13. Seeberger R, Kane G, Hoffmann J, Eggers G (2012) Accuracy assessment for navigated maxillo-facial surgery using an electromagnetic tracking device. J Craniomaxillofac Surg 40: 156-1561. [Crossref]

14. Zheng G, Nolte LP (2015) Computer-Assisted Orthopedic Surgery: Current State and Future Perspective. Front Surg 2: 66. [Crossref]

15. Komune N, Matsushima K, Matsuo S, Safavi-Abbasi S, Matsumoto N, et al. (2017) The accuracy of an electromagnetic navigation system in lateral skull base approaches. Laryngoscope 127: 450-459. [Crossref]

16. Pfisterer WK, Papadopoulos S, Drumm DA, Smith K, Preul MC (2008) Fiducial versus nonfiducial neuronavigation registration assessment and considerations of accuracy. Neurosurgery 62: S201-S207; discussion S7-S8. [Crossref]

17. Guler O, Perwog M, Kral F, Schwarm F, Bardosi ZR, et al. (2013) Quantitative error analysis for computer assisted navigation: a feasibility study. Med Phys 40: 021910 . [Crossref]

18. Norusis M (2003) SPSS 14.0 Statistical Procedures Companion. Upper Saddle River, NJ: Prentice Hall, Inc.

19. Bardosi Z, Freysinger W (2016) Estimating [Formula: see text] distributions of manua fiducial localization in CT images. Int J Comput Assist Radiol Surg 11: 1043-1049. [Crossref]

20. Perwög M, Bardosi Z, Freysinger W (2018) Experimental validation of predicted application accuracies for computer-assisted (CAS) intraoperative navigation with paired-point registration. Int J Comput Assist Radiol Surg 13: 425-441. [Crossref]

21. Cartellieri M, Vorbeck F (2000) Endoscopic sinus surgery using intraoperative computed tomography imaging for updating a three-dimensional navigation system. Laryngoscope 110: 292-296. [Crossref]

22. Soteriou E, Grauvogel J, Laszig R, Grauvogel TD (2016) Prospects and limitations of different registration modalities in electromagnetic ENT navigation. Eur Arch Otorhinolaryngol 273: 3979-3986. [Crossref]

23. Mert A, Gan LS, Knosp E, Sutherland GR, Wolfsberger S (2013) Advanced cranial navigation. Neurosurgery 72: S43-S53. [Crossref]

24. Li L, Yang J, Chu Y, Wu W, Xue J, et al. (2016) A Novel Augmented Reality Navigation System for Endoscopic Sinus and Skull Base Surgery: A Feasibility Study. PloS one 11: e0146996. [Crossref]

25. Ledderose GJ, Hagedorn H, Spiegl K, Leunig A, Stelter K (2012) Image guided surgery of the lateral skull base: testing a new dental splint registration device. Comput Aided Surg 17: 13-20.

26. Mascott CR, Sol JC, Bousquet P, Lagarrigue J, Lazorthes Y, et al. (2006) Quantification of true in vivo (application) accuracy in cranial image-guided surgery: influence of mode of patient registration. Neurosurgery 59: ONS146-ONS156; discussion ONS-56. [Crossref]

27. Ahmadian A, Fathi Kazerooni A, Mohagheghi S, Amini Khoiy K, Sadr Hosseini M (2014) A region-based anatomical landmark configuration for sinus surgery using image guided navigation system: a phantom-study. J Craniomaxillofac Surg 42: 816824. [Crossref]

28. Kral F, DiFranco M, Puschban J, Hoermann R, Riechelmann H, et al. (2014) A new nasopharyngeal dynamic reference frame improves accuracy in navigated skull base targets. Surg Innov 21: 283-289. [Crossref]

29. Bettschart C, Kruse A, Matthews F, Zemann W, Obwegeser JA, et al. (2012) Pointto-point registration with mandibulo-maxillary splint in open and closed jaw position. Evaluation of registration accuracy for computer-aided surgery of the mandible. $J$ Craniomaxillofac Surg 40: 592-598. [Crossref]

30. Chang CM, Jaw FS, Lo WC, Fang KM, Cheng PW (2016) Three-dimensional analysis of the accuracy of optic and electromagnetic navigation systems using surface registration in live endoscopic sinus surgery. Rhinology 54: 88-94. [Crossref]

31. Murbe D, Huttenbrink KB, Zahnert T, Vogel U, Tassabehji M, et al. (2001) Tremor in otosurgery: influence of physical strain on hand steadiness. Otol Neurotol 22: 672-677. [Crossref] 
32. Dorion D, Darveau S (2013) Do micropauses prevent surgeon's fatigue and loss of accuracy associated with prolonged surgery? An experimental prospective study. Ann Surg 257: 256-259. [Crossref]
33. Chang CM, Fang KM, Huang TW, Wang CT, Cheng PW (2013) Three-dimensional analysis of the surface registration accuracy of electromagnetic navigation systems in live endoscopic sinus surgery. Rhinology 51: 343-348. [Crossref]

Copyright: (C2018 Runge A. This is an open-access article distributed under the terms of the Creative Commons Attribution License, which permits unrestricted use, distribution, and reproduction in any medium, provided the original author and source are credited. 